\title{
New realization of Chua's circuit and verification of chaos by harmonic balance analysis
}

Received: 30 September 1997/Accepted: 3 February 1998

\begin{abstract}
Chua's circuit, which consists of two capacitors, one inductor, two resistors, one linear voltage-controlled voltage source (VCVS) and voltage-controlled current source (VCCS) type non-linear element has been realized by operational amplifiers. Various dynamical phenomena such as periodic orbit with periods $T, 2 T$, and $4 T$, single scroll and double scroll similar to the Lorenz attractor have been observed experimentally by changing only the resistance value of the linear resistor $R_{6}$. The experimental observations have been confirmed by computer simulations and also using harmonic balance analysis.
\end{abstract}

Key words Choas $\cdot$ Chua's $\cdot$ circuit .

Harmonic balance analysis

\section{Introduction}

With the new realization of the individual Chua circuit presented in this paper, where the inductor in the Chua circuit of Matsumoto (1985) is also implemented by opapms as in Morgül (1995), the VLSI implementation of the chaotic cellular neural networks of Chua's circuit introduced in Kavaslar and Güzeliş (1995) would be possible. The proposed circuit model shown in Fig. 1, in which

M. E. Yalçin $(\bowtie)$

Electrical-Electronics Engineering Faculty,

İstanbul Technical University,

Maslak TR-80626, Turkey

Tel: +90212 2853618 ;

fax: +90 212285 3679; e-mail: mey@ehb.itu.edu.tr

\section{F. A. Savacı}

İzmir Institute of Technology,

Electrical-Electronics Engineering Dept, Çankaya,

İzmir, Turkey

Tel: + 90232445 1724;

fax: +90 232483 9598; e-mail: savaci@likya.iyte.edu.tr
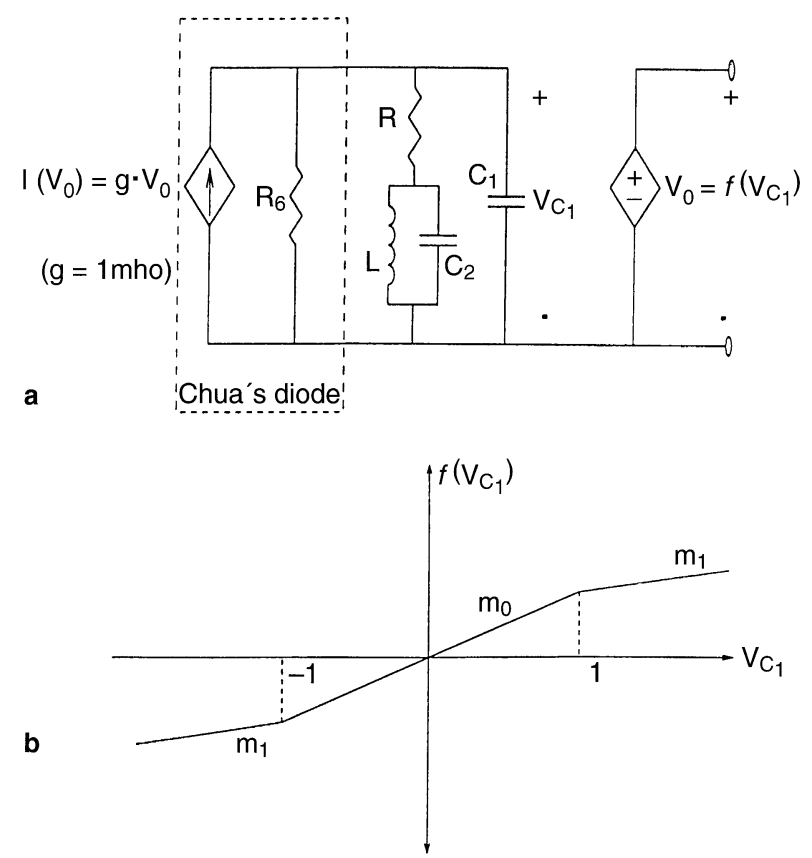

Fig. 1 a VCVS-type realization of Chua's circuit. b The characteristic of piecewise linear VCVS

parallel combination of the linear resistor $R_{6}$ and the voltage-controlled current source (VCCS) is equivalent to Chua's diode (Kennedy 1993), with its non-linear voltagecontrolled voltage source (VCVS) characteristics as in Eq. 3 and with its CNN like topology also allows inhibitory connections as well as excitatory connections. Since the chaotic CNN offers rich dynamical behaviour, such as single-scroll and double-scroll regimes, some pattern recognition applications would be possible, as in Kavaslar and Güzeliş (1995).

In the next sections we give a new realization of Chua's circuit with our experimental results. These results and the simulations have been confirmed by the spectral techniques given in Genesio et al. (1993). Our observation of 
the double scroll also supports the conjecture made in Genesio and Tesi (1992) and Genesio et al. (1993) to predict chaos.

\section{Realization and some experimental observations}

The proposed circuit shown in Fig. 1 has the following corresponding state equations

$\frac{d X}{d \tau}=\mathbf{A} \cdot X+\mathbf{b} \cdot f(y(\tau))$

$y=\mathbf{C} \cdot X$

$f(x)=m_{1} x+\frac{1}{2} \cdot\left(m_{0}-m_{1}\right) \cdot\left(\left|x+E_{1}\right|-\left|x-E_{1}\right|\right)$ where

$\mathbf{A}=\left[\begin{array}{crc}-\alpha(1+\delta) & \alpha & 0 \\ 1 & -1 & 1 \\ 0 & -\beta & 0\end{array}\right]$,

$\mathbf{b}=\left[\begin{array}{l}\alpha \\ 0 \\ 0\end{array}\right], \quad \mathbf{C}=\left[\begin{array}{lll}1 & 0 & 0\end{array}\right], \quad \tau=\frac{t}{C_{2} R_{6}}$,

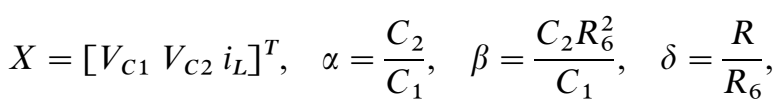

$m_{0}=\frac{\bar{m}_{0}}{R_{6}}, \quad m_{1}=\frac{\bar{m}_{1}}{R_{6}}$.

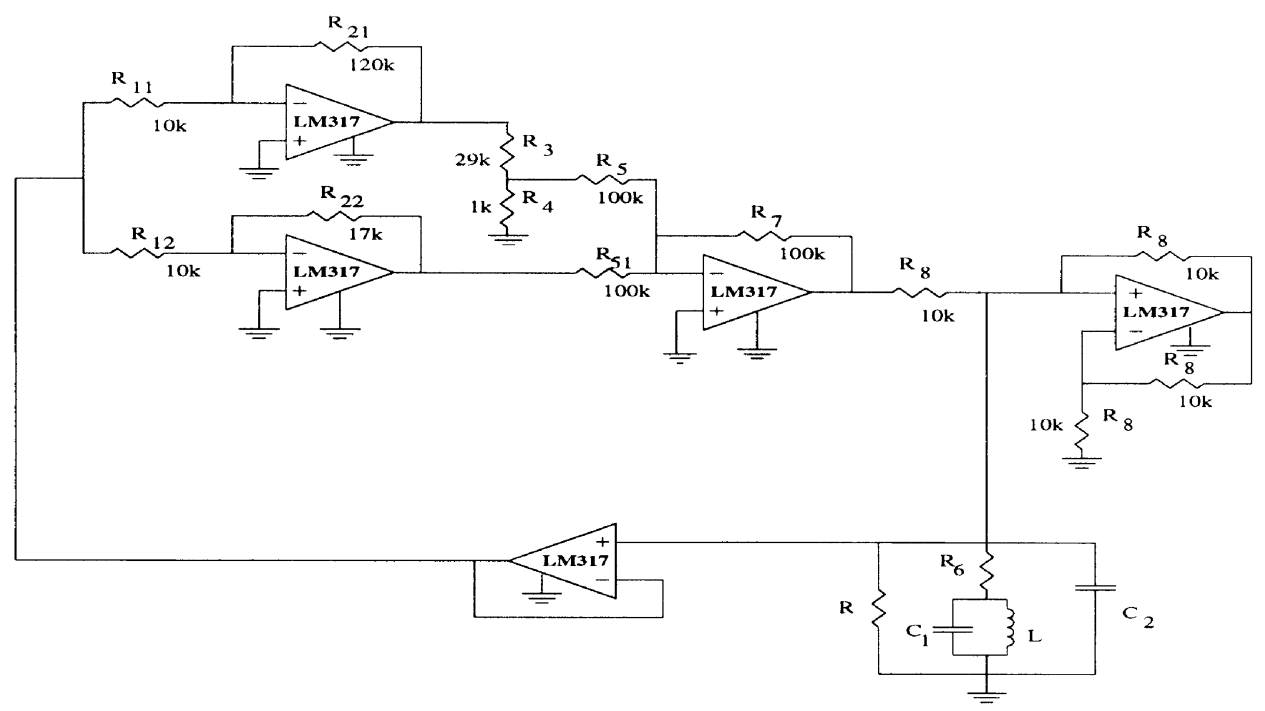

Fig. 2 Implemented circuit $\left(C_{1}=100 n F, C_{2}=1 n F, L=68 m H, R=10 k \Omega, \bar{m}_{0}=\frac{15}{7}, \bar{m}_{1}=\frac{12}{7}, E_{1}=1 \mathrm{~V}\right)$
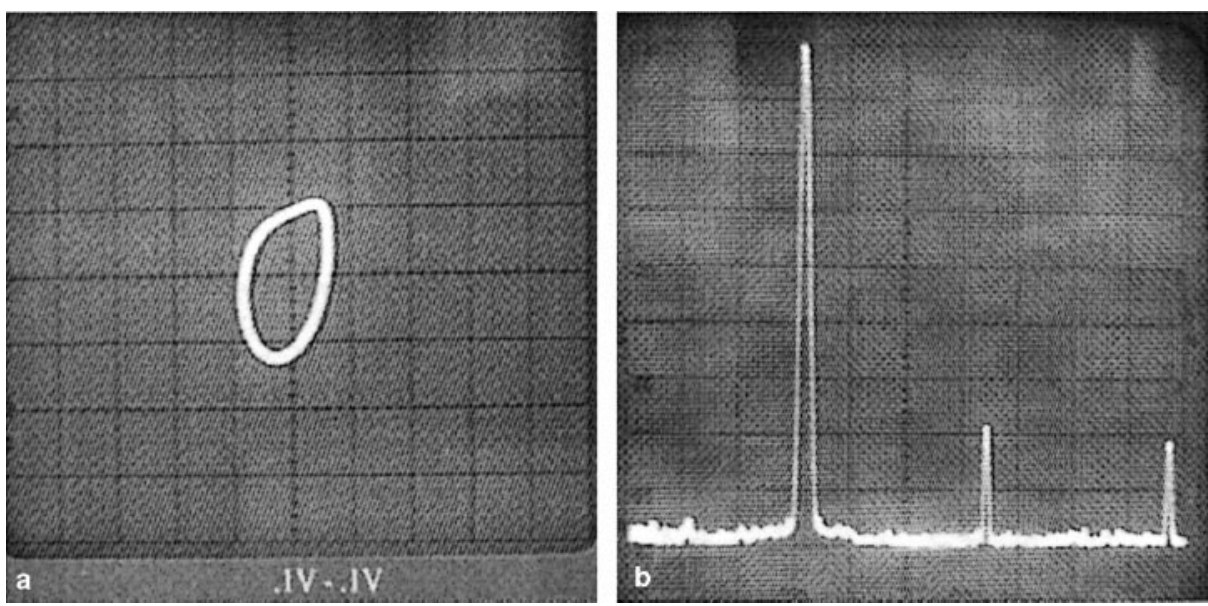

Fig. 3 a Periodic orbit with period $T$ observed with the circuit of Fig. 2 on the $V_{C 1}-V_{C 2}$ plane. Horizontal scale $0.1 \mathrm{~V} /$ div, vertical scale $0.1 \mathrm{~V} /$ div. b Power spectra of the capacitor $V_{C 1}$ on $P_{V_{C 1}}(f)-f$ for period $T$ 
The implemented circuit is shown in Fig. 2, where the bifurcation parameter is chosen to be $R_{6}$ as given in Yalçın et al. (1996). With this implementation the slopes and break points of the characteristic can be independently adjusted, as in Kennedy (1993), the slopes and the breakpoints are $\bar{m}_{0}=\frac{R_{21}}{R_{11}} \frac{R_{4}}{R_{4}+R_{3}}+\frac{R_{22}}{R_{12}}, \quad \bar{m}_{1}=\frac{R_{22}}{R_{12}} \quad$ and $E_{1}=\frac{R_{11}}{R_{21}} E_{\text {sat }}$. By adjusting the value of $R_{6}$ in the circuit of Fig. 2, we have observed periodic orbit with period $T$ on $V_{C 1}-V_{C 2}$ plane for the resistor $R_{6}$ in the range of $7870 \Omega$ to $8217 \Omega$, periodic orbit with period $2 T$ for the range of $8218 \Omega$ to $8277 \Omega$, periodic orbit with period $4 T$ for the range of $8278 \Omega$ to $8291 \Omega$, double-scroll orbit for the range of $8400 \Omega$ to $10400 \Omega$. In Figs 3-6, periodic orbit with periods $T, 2 T$ and $4 T$, double-scroll orbit and their spectra have been given. The observed power spectra confirm a period-doubling route to chaos first discovered by Feigenbaum in the parameter range $0.806<\delta<0.9$, and the first period doubling is a leading indicator for the existence and the nearness of chaotic regions in the parameter space.

\section{Frequency domain analysis}

From the state equations in given by Eqs. 1 and 2 and assuming the system output of the form

$y(t)=A+B \sin (w t)$

and the corresponding non-linearity output

$f(y(t))=N_{0}(A, B) \cdot A+N_{1}(A, B) \cdot B \cdot \sin (w t)$

and then using the well-known harmonic balance method, the frequency domain equations have been obtained as
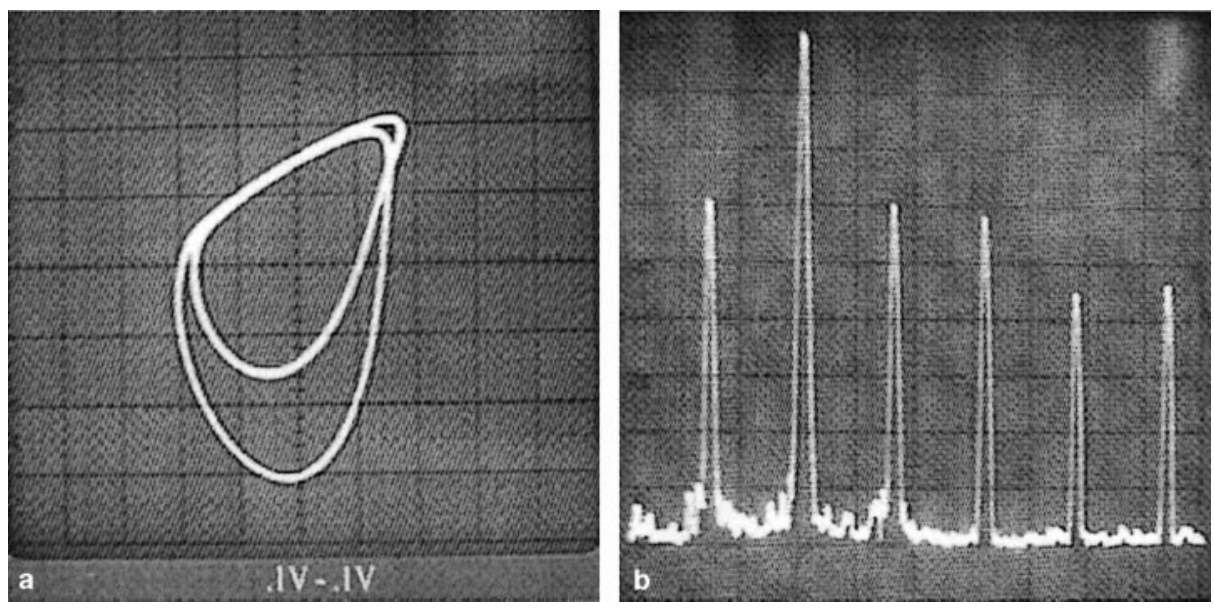

Fig. 4a,b As for Fig. 3 but with period $2 T$
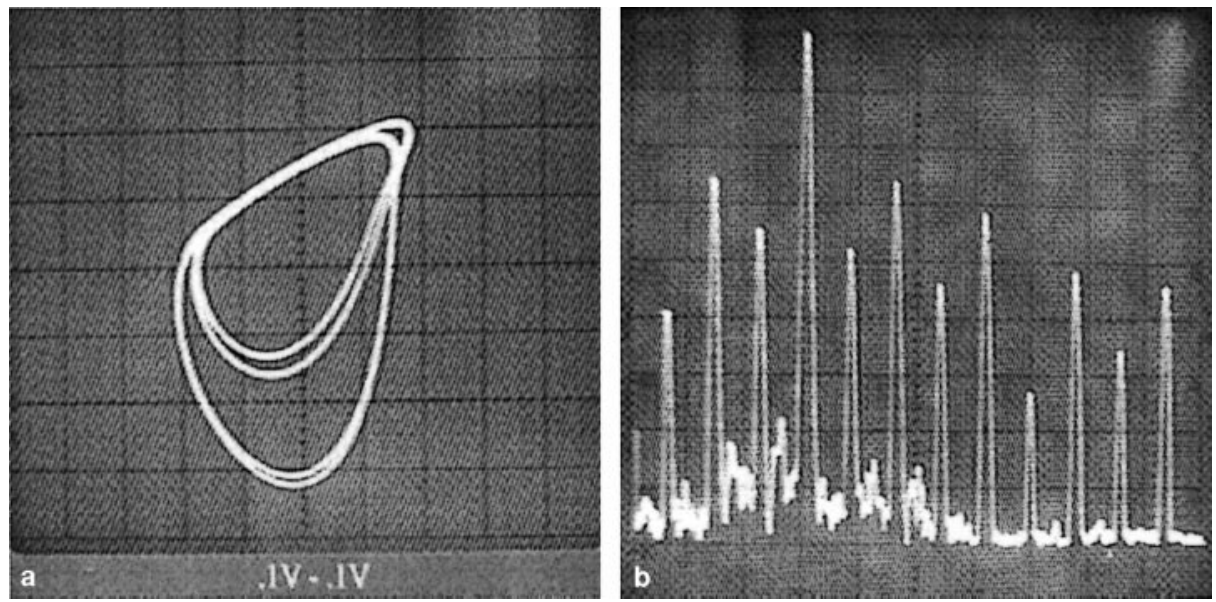

Fig. 5a,b As for Fig. 3 but with period $4 T$ 

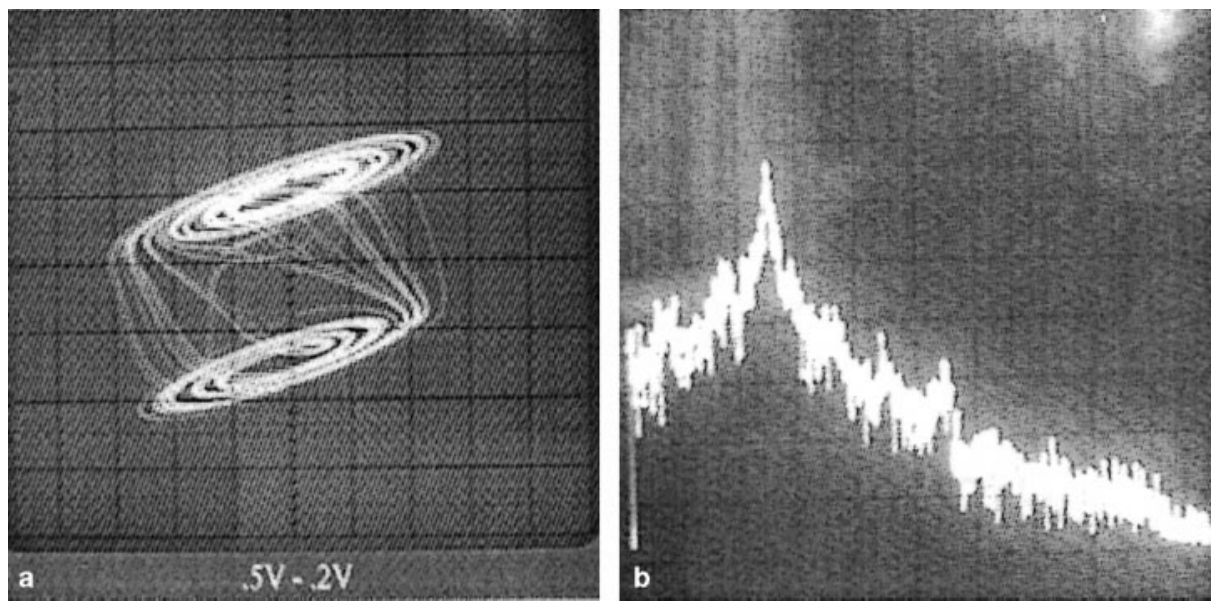

Fig. 6 a Double scroll observed with the circuit of Fig. 2 on the $V_{C 1}-V_{C 2}$ plane. Horizontal scale $2 \mathrm{~V} /$ div, vertical scale $5 \mathrm{~V} /$ div. b Power spectra of the capacitor $\left.V_{C 1}\right)$ on $P_{V_{c 1}}(f)-f$ for double scroll

\section{follows}

$$
\begin{aligned}
& A\left(1-N_{0}(A, B) L(0)\right)=0 \\
& 1-N_{1}(A, B) L(j w)=0
\end{aligned}
$$

where the frequency response of the linear part of the circuit

$$
\begin{aligned}
& L(j w)=C(j w I-A)^{-1} B \\
& \quad=\frac{\alpha\left(\left(\beta-w^{2}\right)+j w\right)}{\left[\alpha \beta(1+\delta)-(1-\alpha(1+\delta)) w^{2}\right]+\left[(\beta+\alpha \delta) w-w^{3}\right] j}
\end{aligned}
$$

and the dual-input describing functions

$$
\begin{aligned}
& N_{0}(A, B)=\frac{\left(m_{0}-m_{1}\right)(1-A) \sin ^{-1}\left(\frac{1-A}{B}\right)}{\pi A} \\
& +\frac{\left(m_{0}-m_{1}\right) B \sqrt{1-\left(\frac{1-A}{B}\right)^{2}}+\frac{\pi}{2}\left(m_{0}-m_{1}+A\left(m_{0}+m_{1}\right)\right)}{\pi A}
\end{aligned}
$$

and

$$
\begin{aligned}
N_{1}(A, B)= & \frac{\left(m_{0}-m_{1}\right)(1-A) \sqrt{1-\left(\frac{1-A}{B}\right)^{2}}}{\pi B} \\
& +\frac{0.5 B \pi\left(m_{0}+m_{1}\right)+B\left(m_{1}-m_{0}\right) \sin ^{-1}\left(\frac{1-A}{B}\right)}{\pi B}
\end{aligned}
$$

With the foregoing equations and using plots of $L(j w)$ and $\frac{1}{N_{1}(A(B), B)}$, experimental observations and simulation results have been confirmed for the following cases in which the stability of the limit cycles has been decided according to the Loeb criterion in Atherton (1975). This is a wellknown method which considers Eq. 7 where $N_{1}(A, B)$ is expressed as function of $B$ from Eq. 6 . Then the stability of the limit cycle depends on the features of the point where the loci of $L(j w)$ and of $1 / N(A(B), B)$ intersect in the Nyquist plane and on the way in which this occurs. Figures 7-9 have been obtained by simulations and

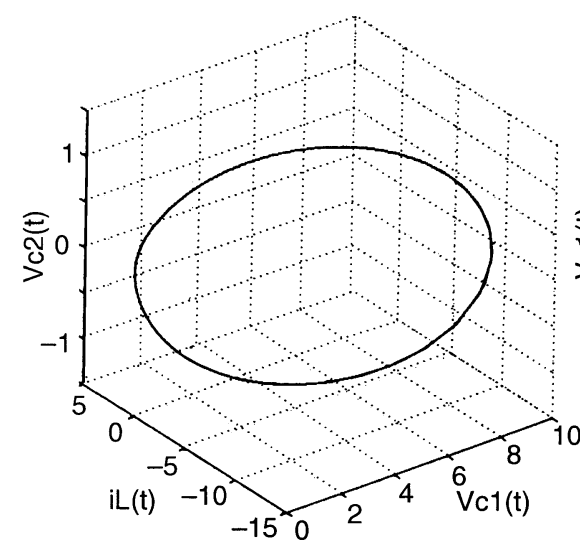

a

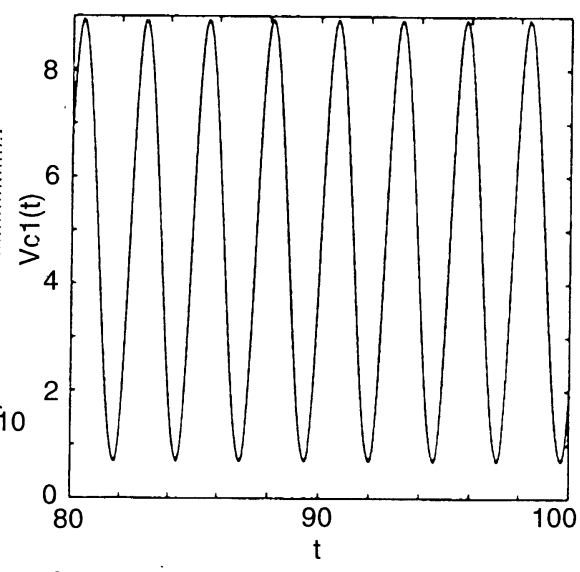

b

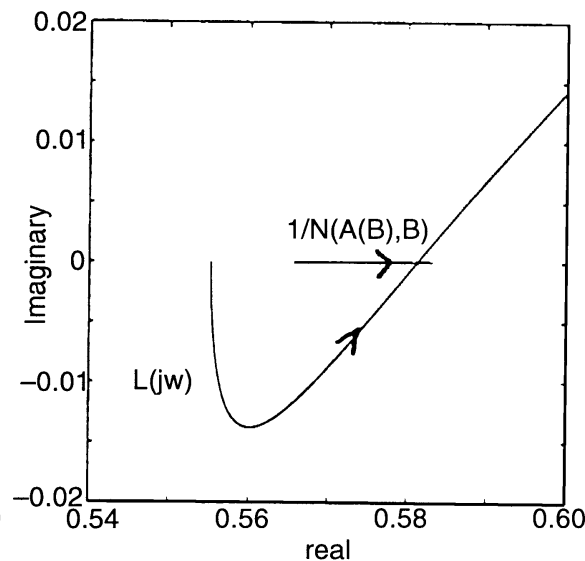

C

Fig. 7a-c For period $T$ : a the phase portrait $\left(V_{C 1}-V_{C 2}-i_{L}\right.$ plane); b $V_{C 1}(t)$ versus $t$; $\mathbf{c}$ Nyquist plots of $L(j w)$ and $\frac{1}{N(A(B), B}$ 


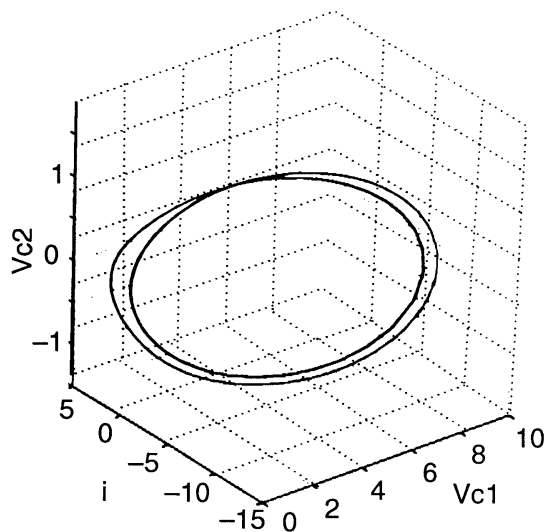

a

Fig. 8a-c As Fig. 7 for period $2 T$

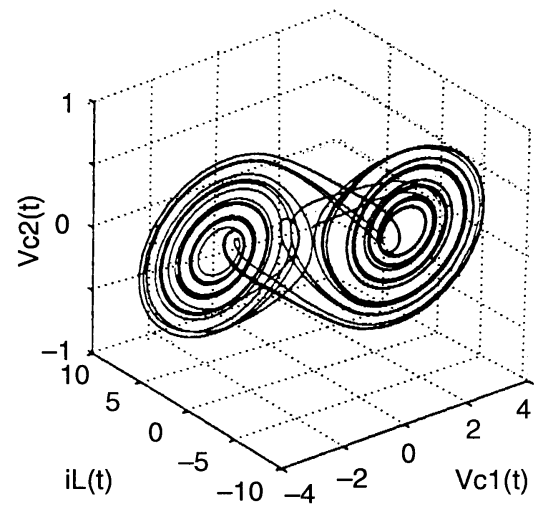

a

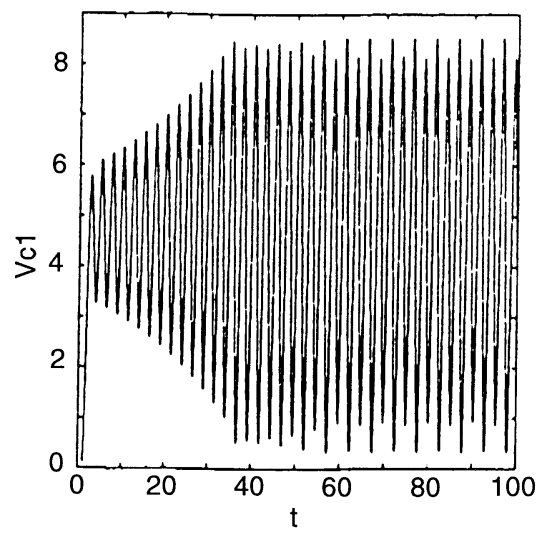

b

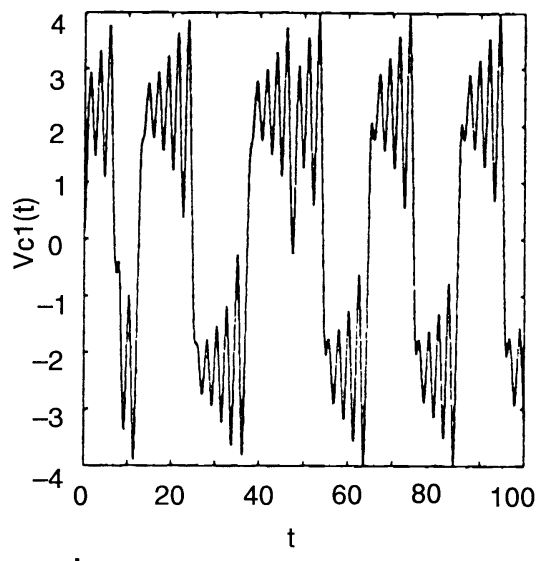

b

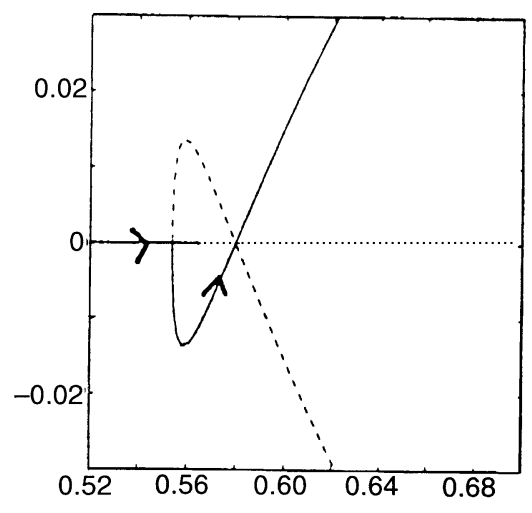

c

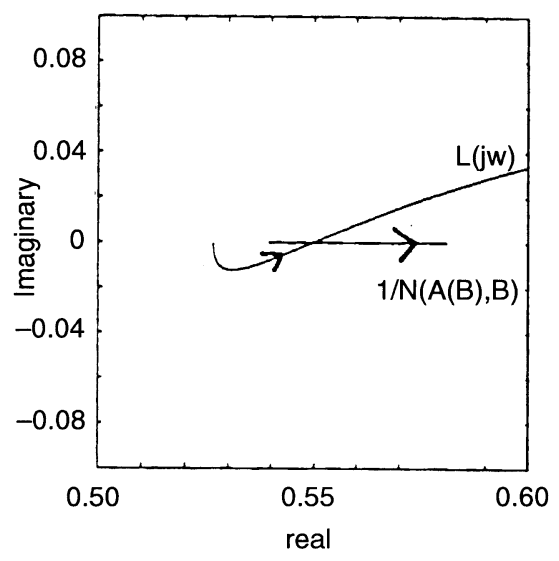

c

Fig. 9a-c As Fig. 7 for double scroll

indicate stable limit cycles. (The motion of the loci of $L(j w)$ with increasing frequency and the motion of the $1 / N(A(B), B)$ with increasing the amplitude $B$ is shown by arrows).

Periodic orbit with period $T$ for $\alpha=9, \beta=14.28$, $\delta=0.801$. From Fig. 7c, the intersection point corresponds to $A=4.8, B=-4.1$ and $w=2.45 \mathrm{rad} / \mathrm{sn}$. Since for these values the stable limit cycle $y(t)$ is not interacting with the unstable equilibrium point 'the origin', chaotic behaviour is not expected, as the conjecture of Genesio and Tesi (1992) and Genesio et al. (1993) suggests.

Periodic orbit with period $2 T$ for $\alpha=9, \beta=14.28$, $\delta=0.806$. In Fig. 8c the intersection point corresponds to $A=4.8, B=-4.0$ and $w=2.45 \mathrm{rad} / \mathrm{sn}$. Once again, for these values the stable limit cycle $y(t)$ is not interacting with the unstable equilibrium point, 'the origin', and so chaotic behaviour is not expected.

Double-scroll behaviour for $\alpha=9, \beta=14.28, \delta=0.900$ : In Fig. 9c the intersection point corresponds to $A=2.1$, $B=-2.7$ and $w=2.5 \mathrm{rad} / \mathrm{sn}$. For these values, the stable limit cycle $y(t)$ and the other symmetrically located stable limit cycle are interacting with the unstable equilibrium point, 'the origin', and since $L(j w)$ satisfies the medium filtering effect, chaotic behaviour has been observed and hence the conjecture given in Genesio et al. (1993) is supported. For this case we have also computed the largest Liapunov exponent as 0.2428 using the standard QR-algorithm (Eckmann et al. 1986) which confirms the chaotic trajectories in Figs 6a and 9a.

\section{Conclusion}

In a similar way to the realizations in Kennedy (1993) using synthesis techniques, we have given op-amp implementations for the piecewise-linear voltage-controlled voltage source in the chaotic CNN. Various dynamical phenomena as shown in Figs 3a-6a and a chaotic spectrum which has a continuous, broad-band nature (Fig. 6b) have been observed. The experimental observations and 
theoretical results based on the harmonic balance analysis have justified the simple model for predicting the existence of chaotic motion given in Genesio and Tesi (1992) and Genesio et al. (1993).

\section{References}

Atherton DP (1975) Nonlinear control engineering. Van Nostrand Reinhold, London

Eckmann JP, Ruelle D, Ciliberto S, Kamphorst SO (1986) Liapunov exponents from time-series. Phys Rev A 34; 4971-4979

Genesio R, Tesi A (1992) Harmonic balance methods for the analysis of chaotic dynamics in nonlinear systems. Automatica 28: 531-548
Genesio R, Tesi A, Villoresi F (1993) A frequency approach for analyzing and controlling chaos in nonlinear circuits. IEEE Trans Circuits Syst 40: 819-829

Kavaslar F, Güzeliş C (1995) A computer-assisted investigatons of a 2D array of Chua's circuits. IEEE Trans Circuits Syst 42: 721-735

Kennedy MP (1993) Three steps to chaos-part II: a Chua's circuit primer. IEEE Trans Circuits Syst 40: 657-674

Matsumoto T, Chua LO, Komuro M (1985) The double scroll. IEEE Trans Circuits Syst 32: 797-818

Morgül Ö (1985) Inductorless realisation of Chua oscillator. Elect Lett 31: 1403-1404

Yalçın ME, Şavkay L, Savacı FA (1996) New realization of Chua's circuit by using voltage-controlled voltage source (VCVS). In: Rodriguez-Vazquez Proc 4th Int Worksh Nonlinear dynamics of electronic systems, IEEE, Seville, pp 259-264 